\title{
A Review of 'Nutrition and Feeding of Organic Poultry 2nd Edition'
}

Published: 3 December 2018

Keywords: diet; feedstuffs; health; organic agriculture; productivity; regulations; requirement; welfare

Nutrition and Feeding of Organic Poultry

Blair $\mathrm{R}$

CABI, Oxfordshire, UK. 2018

278 pp.; ISBN: 978-1786392985

"Nutrition and Feeding of Organic Poultry" is a classical text book, covering a wide range of relevant topics and supported by many literature sources. The author highlights the background of organic poultry production, particularly the growing market with an increase in demand. With feed costs being a main driver of production costs, the economic challenges form the focus point of the introductory section of the book. This is followed by a brief discussion of the principles of organic poultry production and its specific framework. An in-depth discussion of the basic challenge of integrating poultry species into organic farming systems could have led to a more holistic or systemic perspective, but is not explicitly addressed in this section. An interesting overview of the various standards present in different regions worldwide allows for a differentiated perception of "the organic standards" and provides-partially quite detailed-information on the origin and state of regulations in different countries.

Readers without much a priori knowledge on (poultry) nutrition will benefit from the chapter about the basics of poultry nutrition and will be provided an insight into the physiological functioning of poultry. This will allow them to better understand the subsequent specific challenges of organic feeding strategies. The nutrient and energy requirements of the most important poultry species are described in quite some detail, which will make it possible to estimate and analyse the specific needs of birds which will have to be covered by optimised compound feeds.
The individual components from organic origin which are used for producing such feeds are described in quite some detail in one of the core chapters of this book. The information provided enables the reader to achieve a good understanding of the characteristics of the individual feed components which affect their utilization in organic diets. The description of the different traits of feedstuffs, such as nutrient and energy density, content of secondary plant constituents, availability of nutrients, their acceptance by poultry, etc. grants a good understanding of the overall feeding value of a wide range of different feed resources. Despite its inherent focus on North America, the regional differentiation in the presentation of the feedstuffs makes the provided information widely applicable. The author lists a multiplicity of potential feed ingredients and emphasizes the need to critically check the options for their legal use in organic diets in a given country with certification bodies or other authorities. The many experimental studies cited for most of the feedstuffs give a good impression on their scientific evaluation, although it is not always clear how relevant the presented results are in an organic context, i.e. at moderate levels of performance, with the use of different genotypes and under the conditions of free range husbandry systems (which will be briefly discussed in the final chapter). Similarly, the reader is wondering whether some of the feed additives listed here may be legally used in the different countries. However, the core chapter on feedstuffs provides an amazing wealth of information including a comprehensive collection of tables with feeding values for all kinds of feedstuffs. This makes the book not only interesting to read, but to a reference for everybody who wants to deal with the practical nutrition of organic poultry.

The challenge of different practices being allowed in some countries, but not in others, becomes obvious in the chapter on practical feed formulation. Example diets are 
presented for different scenarios, which allow the reader to develop a good feeling for nutritional limitations which may occur, depending on the limitations in place. The author provides comprehensive information on suggested dietary specifications and feed formulas for chickens, turkeys, water fowl, pheasants and game birds, and ratites. The text sections are accompanied by many tables, for which the sequence of diets, premixes and requirements is sometimes a bit confusing. The basics of diet formulation are complemented by statements about type of processing, its consequences on aspects such as particle size and other practically relevant characteristics.

Especially in organic farming, any nutritional measure will strongly depend on the genotype, i.e. breed, strain, hybrid chosen. The author covers this highly relevant aspect for different poultry species and thereby adds to an "organic perspective" that could be expected, but is not always met in textbooks of this kind. Information from different countries allow for a wider view on this thematic field and makes this chapter very interesting to read.

In a final chapter on the integration of feeding programmes into organic production systems a more holis- tic perspective is presented which relates feeding to husbandry and genotype and discusses their potential interactions in terms of the impact on performance, health and welfare of poultry. Topics which are specifically relevant for organic farming, such as the utilization of forage and pasture and specific challenges in organic flocks are presented and add to the value of the book for readers specifically interested in the practical aspects of organic poultry production. In conclusion, "Nutrition and Feeding of Organic Poultry" is a reference book for producers, advisory personnel, teachers, students and technical experts who are searching for sound information on the basics of nutrition, feed characteristics, practical diet formulation and the impact of nutrition on productivity, health and welfare of organic poultry.

Werner J. Zollitsch

BOKU-University of Natural Resources and Life Sciences Vienna, Austria; E-Mail: werner.zollitsch@boku.ac.at 